COMUNICACIÓN CORTA

\title{
Impactación ruminal y abomasal en vacas de cría asociada al consumo de una pastura diferida de Megathyrsus maximus cv. Gatton
}

\author{
Avellaneda-Cáceres $\mathrm{A}^{1^{*}}$, Navarro JA ${ }^{2,3}$, Micheloud JF $F^{1,4}$ \\ ${ }^{1}$ Cátedra de Práctica Hospitalaria de Grandes Animales, Universidad Católica de Salta, Argentina. \\ ${ }^{2}$ Grupo de Producción Animal, Instituto Nacional de Tecnología Agropecuaria (INTA), Salta, \\ Argentina. \\ ${ }^{3}$ Cátedra de Forrajes, Facultad de Ciencias Naturales, Universidad Nacional de Salta, Argentina. \\ ${ }^{4}$ Grupo de Trabajo de Patología, Epidemiología e Investigación Diagnóstica. Área de Sanidad \\ Animal - IIACS Salta, INTA, Salta, Argentina.
}

\footnotetext{
* Correspondencia: Agustín Avellaneda-Cáceres. Facultad de Ciencias Agrarias y Veterinarias, UCASAL, Campus Castañares, 4400 Salta, Argentina.

E-mail: agustin avellaneda 10@hotmail.com
}

Recibido: 28 de Marzo 2019. Aceptado: 30 Abril 2019. Disponible en línea: 14 Mayo 2019

Editor: P. Beldomenico

RESUMEN. La impactación ruminal y abomasal es una afección de los rumiantes que se produce cuando estos consumen un alimento de muy baja digestibilidad y bajo contenido proteico y energético. En este trabajo, se describe una mortandad debido a esta afección, en vacas de cría en el Noroeste argentino. La pastura donde los animales permanecían correspondía a Megathyrsus maximus cv. Gatton, vulgarmente más conocido como "Gatton Panic". Los signos clínicos fueron anorexia, pérdida de estado hasta que los animales cayeron sin posibilidades de incorporarse y posteriormente morían. El diagnóstico se confirmó por los antecedentes clínicos, patológicos y epidemiológicos sumados al análisis de la pastura. El cuadro de impactación ruminal y abomasal está bien descripto en la bibliografía, pero ha sido poco reportado en Argentina.

SUMMARY. Ruminal and abomasal impaction in beef cattle associated with the consumption of a deferred Megathyrsus maximus cv. Gatton pasture. Ruminal and abomasal impaction is a pathological condition of ruminants that occurs when they consume a food with very low digestibility, low protein and energy content. Ruminal and abomasal impaction was registered in breeding cows in the Argentina northwest grazing Megathyrsus maximus cv. Gatton. The clinical signs were anorexia, loss of body condition, recumbency and death. The clinical presentation of ruminal and abomasal impaction is well described in the bibliography but reports are scarce in Argentina.

Palabras clave: bovinos, megatérmicas, digestibilidad, indigestión ruminal

Key words: bovine, megathermical, digestibility, ruminal indigestion

La impactación ruminal y abomasal es una afección de los rumiantes que se produce cuando estos consumen alimentos de baja digestibilidad, sobre todo si está finamente particulado y es de bajo contenido proteico y energético (Ashcroff, 1983). En general, la digestibilidad de los forrajes está inversamente relacionada con su contenido de fibra (Rosales y Pinzón; 2005). La facilidad con la que los microorganismos del rumen son capaces de degradar esa fibra, depende de la distribución de las diferentes moléculas (celulosa, hemicelulosa, lignina) dentro del componente fibroso en la planta (Chesson et al., 1983). El consumo prolongado de alimentos de baja digestibilidad hace que las partículas indigestibles comiencen a acumularse en los pre-estómagos y el abomaso de tal forma que producen distensión y parálisis del órgano que se traduce en atonía de los pre-estómagos (Blood et 
al., 1988). En la región del NOA la producción animal en pastizales se encuentra limitada por la calidad del forraje producido (De León, 2010). Estos pastizales están compuestos principalmente por gramíneas de tipo C4 o tropicales, las que se caracterizan por un crecimiento estival, rápida maduración y una con-secuente disminución de su calidad (Kucseva y Balbuena, 2010). Su ciclo de crecimiento comienza al inicio de la primavera y se detiene cuando disminuye la temperatura a fin de otoño (De León, 2004). Duran-te el invierno (por heladas y/o temporada seca), el contenido de proteína y la digestibilidad del pasto disminuye (Balbuena, 2012). A pesar de que es una afección mundialmente conocida, en Argentina son escasas las descripciones de este trastorno. El obje-tivo del trabajo es describir una mortandad en "goteo", debido a impactación ruminal y abomasal, en vacas de cría asociado al consumo de Mega-thyrsus maximus cv. Gatton ("Gatton panic") diferido en el Noroeste argentino.

En el mes de agosto de 2013 el Servicio de Diagnóstico Veterinario Especializado (SDVE) del INTA EEA Salta efectuó un viaje diagnóstico a un establecimiento ganadero de cría ubicado en la localidad de Dragones, departamento General José de San Martín, provincia de Salta, Argentina. El motivo de consulta fue la mortandad en goteo de vacas de cría iniciada en el mes de mayo del mismo año. El establecimiento contaba con unas 3000 vacas y 600 vaquillonas cruzas Brangus y Braford. Durante la visita, se procedió a la revisación clínica del rodeo y de algunos animales afectados. Todos los animales afectados eran hembras adultas en estado avanzado de gestación (6-8 meses). Se encontraban caídos en decúbito esternal y efectuaban infructuosos intentos para levantarse. El estado del sensorio era normal pero finalmente morían entre 4 y 7 días de iniciado el cuadro. A la inspección se observó marcada distensión abdominal derecha, izquierda o ambas (Figura 1a) y a la palpación rectal se identificó incremento de la consistencia del contenido ruminal y dolor que se manifestaba a través de quejidos. A la percusión-auscultación se apreció matidez en la cúpula dorsal del rumen y en el área de proyección abomasal. La alimentación se basaba en el pastoreo de una pastura diferida de $M$. maximus de baja disponibilidad. Todos los corrales contaban con bebederos automáticos. Se colectaron muestras de sangre y del forraje disponible. De las muestras sanguíneas se obtuvieron los valores de calcio (Ca), fósforo $(\mathrm{P})$, magnesio $(\mathrm{Mg})$, proteínas totales (PT), albúmina (Alb), así como la actividad de las enzimas creatina-fosfocinasa (CPK) y aspartato aminotransferasa (AST) (Tabla 1). El forraje fue sometido a análisis de materia seca $(\mathrm{MS})$, fibra en detergente neutro (FDN), fibra en detergente ácido (FDA), proteína bruta (PB) y nitrógeno total (Tabla 2). El valor de digestibilidad de la materia seca (DMS) se calculó a partir del valor de FDA, utilizando la ecuación de predicción: DMS = 88,9 - $(0,779$ x FDA) propuesta por Rohweder et al. (1978), la cual es la que mejor ajusta para las pasturas tropicales. Finalmente se efectuó la necropsia completa de tres animales que yacían muertos y durante ésta se colectaron muestras de tejidos en formol bufferado al $10 \%$. Las muertes se prolongaron hasta el mes de septiembre, contabilizándose un total de 20 vacas muertas al finalizar el cuadro $(0,6 \%)$. Todos los animales que enfermaron murieron por lo que la letalidad fue del $100 \%$.

Tabla 1: Valores de calcio, fósforo, magnesio, proteínas totales, albumina y actividad de las enzimas: creatina-fosfocinasa (CPK) y aspartato aminotransderasa (AST) en suero. (valores de referencia: Radostits, 2002). Ca=Calcio; $\mathrm{P}=$ =Fosforo; $\mathrm{Mg}=$ Magnesio; $\mathrm{PT}=$ Proteínas Totales; Alb= Albumina; $\mathrm{CPK}=$ Creatina-fosfocinasa; AST=Aspartato aminotransferasa.

\begin{tabular}{|c|c|c|c|c|c|c|c|}
\hline Muestras & $\begin{array}{c}\mathrm{Ca}^{2+} \\
\mathbf{m g} / \mathbf{d L}\end{array}$ & $\begin{array}{c}\mathbf{P} \\
\mathbf{m g} / \mathbf{d L}\end{array}$ & $\begin{array}{c}\mathbf{M g} \\
\mathbf{m g} / \mathbf{d L}\end{array}$ & $\begin{array}{c}\mathbf{P T} \\
\mathbf{g} / \mathbf{d L}\end{array}$ & $\begin{array}{c}\text { Alb } \\
\mathbf{g} / \mathbf{d L}\end{array}$ & $\begin{array}{c}\mathrm{CPK} \\
\mathbf{U} / \mathbf{l}\end{array}$ & $\begin{array}{c}\text { AST } \\
\mathbf{U} / \mathbf{l}\end{array}$ \\
\hline $\mathbf{1}$ & 8,2 & 3,3 & 2,3 & 8,6 & 3,3 & $>1000$ & 850 \\
\hline $\mathbf{2}$ & 9,7 & 2,3 & 3,0 & 7,3 & 2,3 & 946 & 292 \\
\hline Referencias & $9,7-$ & $5,6-$ & $1,8-$ & $5,7-$ & $2,1-$ & $35-$ & $78-$ \\
& 12,4 & 6,5 & 2,3 & 8,1 & 3,6 & 280 & 132 \\
\hline
\end{tabular}

Tabla 2: Valores composicionales de los parámetros nutricionales de Megathyrsus maximus cv. Gatton diferido. MS=Materia Seca; NT =Nitrógeno Total; PT = Proteína Total; F.D.A = Fibra Detergente Acida; F.D.N= Fibra Detergente Neutra; DMS= Digestibilidad de la materia seca; EM= Energía Metabolizable.

\begin{tabular}{|c|c|c|c|}
\hline $\begin{array}{c}\text { MS } \\
(\%)\end{array}$ & $\begin{array}{c}\text { NT } \\
(\%)\end{array}$ & $\begin{array}{c}\text { PT } \\
(\%)\end{array}$ & $\begin{array}{c}\text { F.D.A } \\
\text { (\%) }\end{array}$ \\
\hline 93,2 & 0,90 & 5,62 & 52,70 \\
\hline \begin{tabular}{c|c|c|} 
F.D.N \\
$(\%)$
\end{tabular} & $\begin{array}{c}\text { DMS } \\
(\%)\end{array}$ & $\begin{array}{c}\text { EM } \\
\text { (Mcal Kg/MS) }\end{array}$ \\
\hline 78,70 & 47,85 & 1,48 \\
\hline
\end{tabular}

Durante la necropsia, dos de los cadáveres inspeccionados presentaban marcado timpanismo y dilatación abdominal. A la apertura de la cavidad abdominal se apreció que el rumen presentaba gran distención, su contenido era macizo y estaba constituido por fibras vegetales gruesas (Figura 1b). Al retirar las paredes del órgano, el material permanecía organizado y costaba desarmarlo manualmente. Además, se apreció escasa cantidad de líquido y el olor del licor ruminal era imperceptible. El hígado presentaba reducción del tamaño, aunque se denotaba una marcada repleción de la vesícula biliar. El tracto intestinal se encontraba totalmente vacío. En uno de los cadáveres se observó un franco 
incremento del tamaño del abomaso, el cual presentaba gran cantidad de contenido fibroso similar al descripto para el contenido ruminal. En este último caso el rumen denotaba marcada reducción del tamaño (Figura 1c) y el contenido era similar al de los cadáveres anteriores.

Microscópicamente, en todos los casos evaluados se observó esteatosis hepática microvacuolar y reducción del tamaño (atrofia) de los hepatocitos. Los demás órganos no presentaban lesiones de relevancia diagnóstica.

Se arribó al diagnóstico de impacción de rumen y abomaso contemplando la signología clínica observada, los hallazgos patológicos y bioquímicos y la asociación con los antecedentes del caso. En general los trastornos de impactación de origen primario se deben a la ingestión de grandes cantidades de forraje de baja calidad y puede estar o no asociada a restricción hídrica (Blikslager et al., 1995). Según Rodríguez y Allasia (2011) los cuadros de impactación sobrevienen cuando los forrajes tienen elevados valores de FDN y FDA, y bajo contenido de proteína bruta (menor al 12\%). Coin-cidentemente, en este caso el análisis del forraje arrojó niveles de fibra elevados (FDA: 52,70 \%y FDN: 78.70\%) asociados con bajos niveles de proteína (PB: 5.62\%) y baja digestibilidad (DMS:47,85\%)

En Norteamérica describen cuadros de impactación abomasal en vacas de cría en los meses de invierno debido al consumo de alimentos de baja calidad con poca o nula agua disponible (Hoffsis y McGuirk, 1993; Guard, 2006), siendo mayor la incidencia en vacas en gestación avanzada (Pope, 1961; Merrit \& Boucher, 1967; Blikslager et al., 1995). Coincidiendo con esto, Mitchell (1991) indica que las vacas preñadas en época invernal son más propensas a desarrollar la enfermedad debido al aumento de la demanda orgánica por su estado fisiológico. Esto se observó en este caso donde todos los animales afectados fueron vacas adultas de unos 7 a 8 meses de gestación. Mattioli y Relling (2013) han denominado a este trastorno como "indigestión por rendimiento deficiente de la flora microbiana ruminal" e indican que la reducción en la cantidad de microorganismos totales, reduce la velocidad de fermentación de los alimentos lo cual conduce a una acumulación paulatina de material sin digerir en el rumen.

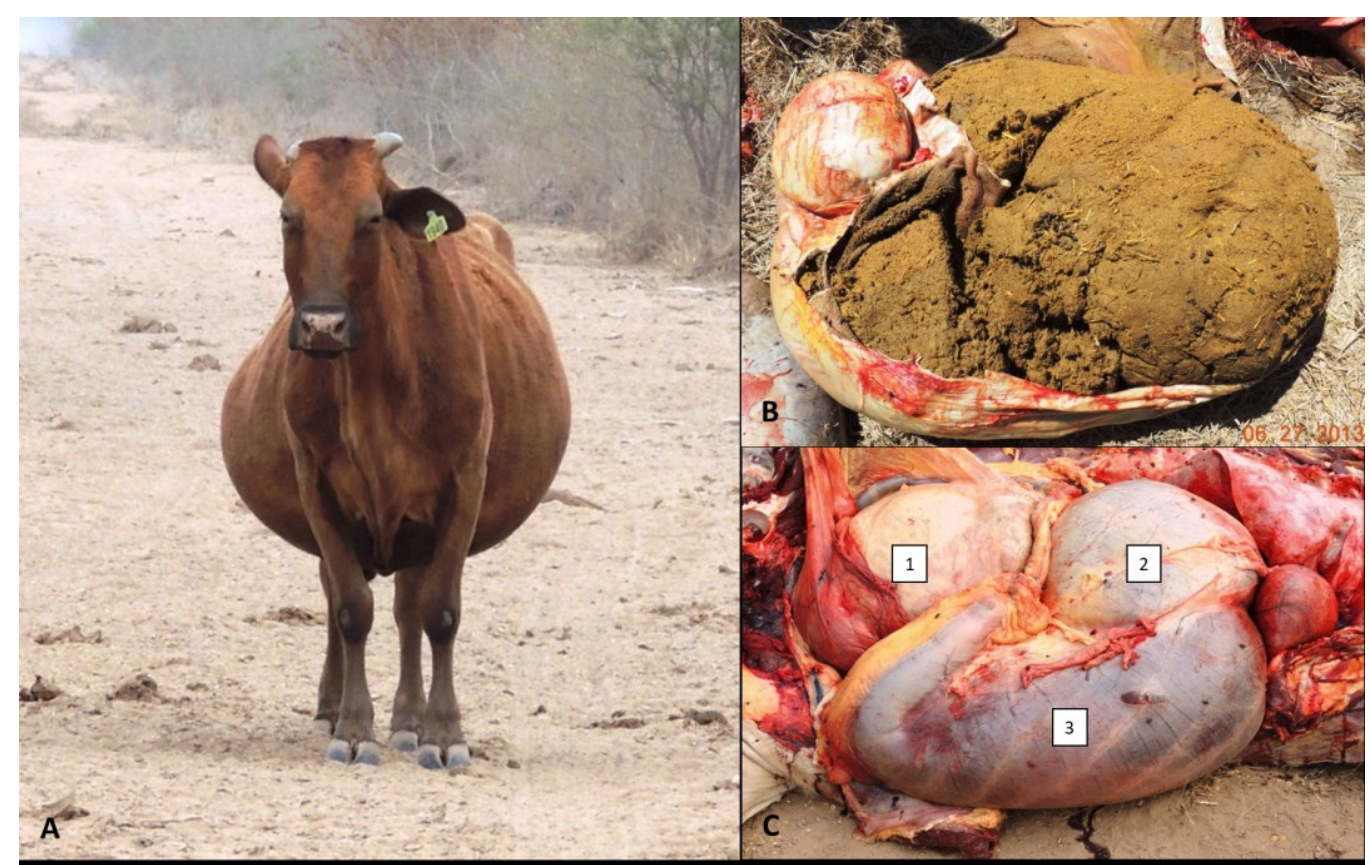

Figura 1: A- Animal afectado - Se puede observar la dilatación ventrolateral derecha (área de proyección abomasal) y dorso-lateral izquierda (área de proyección ruminal). B- Contenido ruminal de consistencia sólida. Al retirarse las paredes del rumen la forma del mismo se mantenía inalterada. C- Intensa distención omasal (2) y abomasal (3) con retracción ruminal (1). A la apertura del órgano se observó la presencia de gran cantidad de material fibroso y finamente particulado en el interior del órgano.

Esta acumulación de material en los pre-estómagos ocasiona atonía, distensión abdominal e incremento de la consistencia de los órganos a la palpación rectal y dolor que se manifiesta a través de quejidos (Blood et al., 1988). Consecuentemente se desarrolla anorexia y pérdida de estado hasta que los 
animales caen sin posibilidades de incorporarse (Buffarini y Tellechea, 2008). La evolución del cuadro depende del grado de impactación y de la gravedad del desequilibrio hidroelectrolítico (Cantón, 2009). Buffarini y Tellechea, (2008) indican que los animales más afectados mueren luego de 3 a 6 días de iniciados los signos clínicos. Estos mismos autores mencionan haber observado peritonitis debido a la perforación del abomaso u alguna otra víscera por el material grosero, pero esto no fue encontrado en los casos aquí observados. La impactación abomasal por su parte es de evolución más aguda y precipita mucho más rápido hacia la muerte (Ashcroff, 1983). Finalmente, aunque sabemos que los recursos forrajeros juegan un papel fundamental en la nutrición de rumiantes no debemos desconocer que, como fuente de nutrientes, presentan una composición muy variable. Por tanto, no toda la fibra vegetal es digerible en el rumen, aun cuando pueda permanecer en él por mucho tiempo. En general, el contenido de lignina de los forrajes ha sido asociado negativamente con la degradabilidad de la fibra y se cree que la lignina actúa como una barrera física a la degradación microbiana de la fibra en el rumen (Rozales y Pinzón, 2005). En Argentina a pesar de que la alimentación de bovinos con forrajes de baja calidad se emplea comúnmente, especialmente en inviernos secos, estos cuadros de impactación ruminal y abomasal han sido poco diagnosticados. En tanto, por la dificultad del diagnóstico, es posible que muchos casos pasen desapercibidos (Borges et al. 2007). Lo que pone en manifiesto que este trastorno puede ser muy frecuente en los rodeos bovinos que son mantenidos sobre pasturas diferidas.

\section{Bibliografía}

Ashcroft RA. 1983. Abomasal impaction of cattle in Saskatchewan. Can. Vet. J. 24: 375:380.

Balbuena O. 2012. Uso de alimentos disponibles en la región NOA y NEA en la alimentación del ganado. Rev. Amanecer Rural 120: 18- 23

Blikslager AT, Anderson KL, Bristol DG, Fubini SL, Anderson DE, 1995. Abomasal impactation in cattle. J. Am. Vet. Med. Assoc. 15: 571-573.

Blood DC, Henderson JA, Radostits OM. 1988. Impactación nutricional del abomaso en los Bovinos. En: Medicina Veterinaria 6. Nueva editorial interamericana. Pp: 276-278.
Borges JRJ, Cunha PHJ, Moscardini ARC, Franco GL, Silva LAF. 2007. Compactação de abomaso em bovinos leiteiros: descrição de cinco casos. Ciência Anim. Bras. 8: 859-864.

Buffarini M y Tellechea DE. 2008. Mortandad en un rodeo de vacas por impactación ruminal. Reporte de un caso. Memoria Técnica 2007-2008, Estación Experimental agropecuaria General Villegas Publicaciones Regionales ISNN 1850-6038. Pp. 78-80.

Cantón G, Odriozola E, Giannitti F, Fernández M, Spinelli $R$, 2009. Impactación de abomaso en vacas de cría. Veterinaria Argentina 25: 651

Chesson A, Gordon AH, Lomax JA. 1983. Substituent Groups Linked by Alkali-labile Bonds to Arabinose and Xylose Residues of Legume, Grass and Cereal Straw Cell Walls and their Fate during Digestion by Rumen Microorganisms. J. Sci. Food Agric. 34: $1330-1340$.

De León M. 2004. Invernada sobre pasturas subtropicales. Informe Técnico N4. Año 2004. EEA Manfredi. Pp. 1-4.

De León M, EEA Manfredi, INTA. 2010. Megatérmicas para mejorar la ganadería subtropical. Planteos ganaderos en siembra directa. Revista técnica de la Asociación Argentina de Productores en Siembra Directa. Pp. 47-53.

Guard C. 2006. Impactação abomasal. En: Smith BP (ed.). Tratado de Medicina Interna de Grandes Animais. 3a edição. Manole, São Paulo. Pp. 763-765.

Hoffsis GF y McGuirk SM. 1993. Abomasal impactions in cattle. En: Howard JL y Smith RA (eds.). Current Veterinary Therapy 3: Food Animal Practice. WB. Saunders Company Ltd., Philadelphia, USA. Pp. 732-733.

Kucseva CD, Balbuena O. 2010. Efectos de la suplementación sobre el consumo de pastos tropicales. Jornadas Proyecto Nacional de Nutrición Animal, Programa Nacional Carnes, Proyecto Integrado Nutrición. ISBN № 978-987-1623-96-9. Pp. 47-57.

Merrit AM y Boucher WB. 1967. Surgical treatment of abomasal impaction in the cow. J. Am. Vet. Med. Assoc. 150: 1115-1120.

Mitchell KJ. 1991. Dietary abomasal impaction in a herd of dairy replacement heifers. J. Am. Vet. Med. Assoc. 198: 1408-1409.

Pope DC. 1961. Abomasal impactation of adult cattle. Vet. Rec. 73: $1174-1176$.

Radostits OM, Gay CC, Blood DC, Hinchcliff KW. 2002. Valores analíticos de referencia - Constituyentes séricos. En: Medicina Veterinaria. 9a Edición. Vol 1. Mcgraw-hill. Madrid, España. Pp. 2160-2161.

Relling AE y Mattioli GA. 2013. Fisiología digestiva y metabólica de los rumiantes. Segunda edición. Ed. CCB Academic Press, Argentina. 106 pp.

Rodríguez RA y Allasia MA. 2011. Algunos trastornos primarios del sector gástrico anterior de los rumiantes. 2da. Edición. Imprenta Macagno S.R.L. Santa Fe, Argentina. Pp. 47-57.

Rohweder DA, Barnes RF, Jorgensen N. 1978. Proposed hay grading standards based on laboratory analyses for evaluating quality. J. Anim. Sci. 47: 747-759. 
Rosales RB y Pinzón SS. 2005. Limitaciones físicas y químicas de la digestibilidad de pastos tropicales y estrategias para aumentarla. Corpoica Ciencia y Tecnología Agropecuaria 6: 69-82. 